\title{
INVESTIGATION OF BEHAVIOUR OF 3 DEGREES OF FREEDOM SYSTEMS FOR TRANSIENT LOADS
}

\author{
J.O.Kiran ${ }^{1}$, D.Shivalingappa ${ }^{2}$ \\ ${ }^{1}$ Assistant Professor, Department of Mechanical Engineering, S.T.J Institute of Technology, Ranebennur, \\ Karnataka, India, kiranjajur@yahoo.com \\ ${ }^{2}$ Professor, Department of Mechanical Engineering, Adhiyamaan College of Engineering, Hosur, \\ Tamilnadu, India,dsivadvg@gmail.com
}

\begin{abstract}
In this work, the energies dissipated by the spring mass damper system with three degrees of freedom are modelled and simulated for three types of external loads, namely, constant load, exponential decaying load overtime and a partial load over a time period. Two models of the spring mass damper system are modelled and the governing equations are derived. The velocities of the oscillators are estimated by solving the corresponding governing equations for loss of factor of 0.15. The kinetic and potential energies are calculated using the mass, velocity and stiffness of the oscillators and total energy is estimated. , when the load is changed from full load to a partial load over a time period, there is significant increase in the displacement and the velocity at near $0.75 \mathrm{sec}$, which means it dissipates more energy The contribution of the kinetic energy is minimal for oscillator 2 in all cases and the total energy is constituted mostly of potential energy and there is a substantial contribution both by kinetic and potential energy of oscillator 1 and 3 is presented in this paper.
\end{abstract}

Index Terms: Vibration, 3 Degrees of freedom, Dampers, Loss factor, Transient loads.

\section{INTRODUCTION}

Applications like automobiles, aeroplanes, space crafts, civil structures engineering materials for building the structures. Most of the applications are dynamic and some are static. Examples of static structures are civil structures and the dynamic applications are the rest of the above list. The engineering components are elastic materials which transfer the energy or motion or load from one component to other component [1,2]. When the applied load acts on the elastic materials, they undergo vibrations. These materials also have some amount of built in damping characteristics which reduces the effect of vibrations. In other words, some part of the energy is utilized in overcoming the internal damping of the system. The vibration has two important characteristics and they are amplitude and frequency of vibration apart from other factors. Amplitude of the vibration becomes very high when there is alternating load acting and the frequency of this load is close enough to matching with the natural frequency of the system. The frequencies at which this kind behaviour is exhibited by the system is known as resonant frequencies [3].

The energy dissipated by the system may be termed as total energy and it consists of two parts, viz. Potential Energy and Kinetic Energy. Potential energy is the one which is dissipated in overcoming the stiffness of the system and the kinetic energy is the energy dissipated due the mass of components of the system and their velocity [4].
For a system with three degrees of freedom, there are three masses connected to each other through elastic stiffeners or springs and dampeners. The configuration of the masses, springs and dampeners must be derived from the actual application and assumptions are made in simplified representation. When the external load is applied on the system, the system undergoes the vibrations. The type of loads acting on the system can be classified as external loads or due to movement of the base excitation or movement of any other component of system. The external loads are of different types, namely, constant load, harmonic load, exponentially decaying loads, pulse loads over a full time period or partial time period etc. The response of the system can be steady state or transient in interest. The potential energy may also be termed as strain energy [5].

The energy input into the system may be either stored inside the system or dissipated. The energy which is dissipated may be termed as loss of energy in technical terms. It is important during any system design to know how much energy may get dissipated and essential to know the amount of loss of energy. The loss factor is required to be estimated for the system in use.

The loss factorn may be defined as the ratio of the dissipated power per radian to the total energy of the structure [6-11].

$\eta=\frac{P_{\text {Disspated }}}{\omega \mathrm{E}_{\text {Total }}}$ 
This can also be written as

$\eta=\frac{P_{\text {Input }}}{\omega \mathrm{E}_{\text {Total }}}$

or

$\eta=\frac{E_{\text {Input }}}{\omega \varepsilon_{\text {Total }}}$

where

$\mathrm{E}_{\text {Input }}:$ Energy input to the structure and

$\varepsilon_{\text {Total }}:$ Total integrated energy

$\varepsilon_{\text {Total }}=\varepsilon_{\text {Kinetic }}+\varepsilon_{\text {Potential }}$.

Power Input Method:

For a structural system, the loss

factor $\eta$ can also be written as

$\eta(\omega)=\frac{\Delta E}{E_{S E}}$

where

$\mathrm{E}_{\mathrm{SE}} \quad$ : Strain energy

$\Delta \mathrm{E} \quad$ : Energy dissipated.

The energy that is input to the system can be obtained by measuring the force and the velocity at the point of input. However, the measurements of force and velocity to be obtained simultaneously. The energy input to the system can also be estimated by [6-9, 10-11]

$\mathrm{E}_{\mathrm{in}}=\frac{1}{2 \omega} \operatorname{Re}\left[\mathrm{h}_{\mathrm{ff}}(\omega)\right] \mathrm{G}_{\mathrm{ff}}(\omega)$

Where

$\mathrm{h}_{\mathrm{ff}} \quad$ : Mobility function of the driving point

$\mathrm{G}_{\mathrm{ff}} \quad$ : Power spectral density of the input force

The strain energy $\mathrm{E}_{\mathrm{SE}}$ can be obtained by measuring the kinetic energy as follows:

$\mathrm{E}_{\mathrm{KE}}=\frac{1}{2} \sum_{\mathrm{i}=1}^{\mathrm{N}} \mathrm{m}_{\mathrm{i}} \mathrm{G}_{\mathrm{ii}}(\omega)$

Where

$\mathrm{E}_{\mathrm{KE}} \quad$ : Kinetic energy of the system

$\mathrm{N}$ : Number of location points where measurements are carried out

$\mathrm{m}_{\mathrm{i}} \quad$ : Mass of discrete locations of the system

$\mathrm{G}_{\mathrm{ii}} \quad$ : Power spectral density of the velocity

If the system is assumed to be linear, then

$\left|h_{i f}(\omega)\right|^{2}=\frac{G_{i i}}{G_{f f}}$

where

$h_{\text {if }} \quad:$ Transfer mobility function

If all the points of measurement are equally spaced and having equal mass portions, Eqs. 5 to 8 can be written as

$\eta(\omega)=\frac{\operatorname{Re}\left[\mathrm{h}_{\mathrm{ff}}(\omega)\right]}{\omega \mathrm{m} \sum_{\mathrm{i}=1}^{\mathrm{N}}\left|h_{i f}(\omega)\right|^{2}}$.

In order to estimate the loss factors accurately, it is essential to have accurate measurements. Otherwise, there is a possibility of making large errors which does not serve the purpose. The power input method requires few numbers of steps than the experimental Statistical Energy Analysis. The loss factors obtained from the power input method can be used in SEA to predict the vibration and for any parametric studies [12-26].

In this section, the basic definitions along with the literature available on the related work are explained. In Sec. II, analytical models are derived along with the applicable governing equations. In Sec. III, the simulation results are presented and the results are discussed. Finally important conclusions are drawn.

\section{ANALYTICAL MODEL}

In this section, two different models with three degree of freedom are simulated for two cases of stiffness and dampers [18-21]. The governing equations are derived and they are represented in the matrix form. By solving the governing equations, the energy stored in form of kinetic energy and potential energy in the model can be estimated.

Figs.1 and 2 shows the spring mass damper system with three degrees of freedom for model 1 and model 2, respectively. In these two cases, $\mathrm{m} 1, \mathrm{~m} 2$ and $\mathrm{m} 3$ represent masses which are connected to four springs of stiffnesses represented by $\mathrm{k} 1, \mathrm{k} 2, \mathrm{k} 3$ and $\mathrm{k} 4$. The configurations of dampeners are different between two models. The dampeners c1, c2, c3 and c4 are attached to the masses as shown in Fig 1 for model 1. In case of model 2,the dampers c3, c4 and c5 are used as shown in Fig. 2. Damper c3 connects only masses $\mathrm{m} 2$ and $\mathrm{m} 3$, and damper c4 connects mass $\mathrm{m} 3$ and the rigid surface and damper $\mathrm{c} 5$ connects mass $\mathrm{m} 1$ and rigid surface. In both the models, the force F2 acts directly on the oscillator 2 .

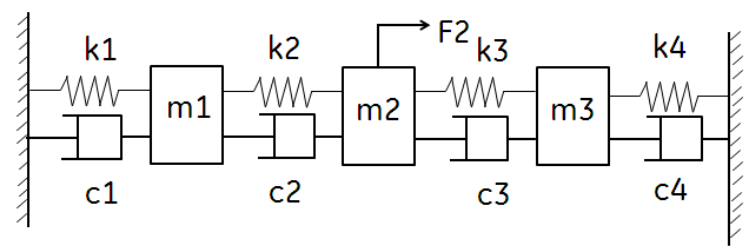

Fig -1: Spring Mass and Damper System of Model 1

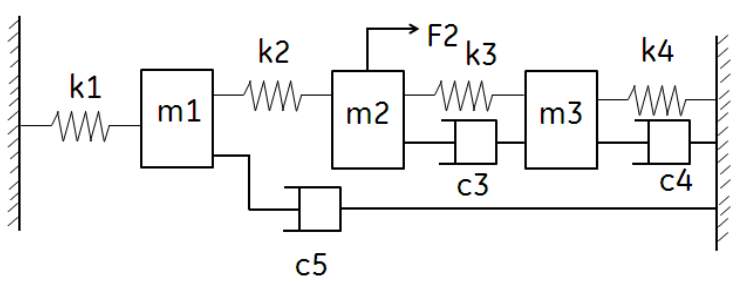

Fig -2: Spring Mass and Damper System of Model 2

However, the nature of the load F2 is varied and the submodels are derived. In first sub model 1a, the load F2 is a constant load for the full time period ( $\mathrm{T}$ sec) and in sub model $1 \mathrm{~b}$, the load F2 is exponentially decaying load for the entire time period. In model 1c, the load F2 is constant for certain duration of the time step and reduces to zero for the remain period of the time step.

$F 2=\overline{F 2}$ for $t=T$ 


$$
F 2=\overline{F 2} e^{-w t} \text { for } t=T
$$

$$
F 2=\overline{F 2} . \delta(t), \ldots . . \delta(t)=\left\{\begin{array}{l}
1 \text { for } t \leq T \\
0 \text { for } t>T
\end{array}\right.
$$

Eqs. 10,11, and 12 represent the loads acting on the oscillator 2 for model $1 \mathrm{a}, 1 \mathrm{~b}$ and $1 \mathrm{c}$ respectively. Similarly, the same loads are repeated for the model 2 and the sub models are named as models $2 \mathrm{a}, 2 \mathrm{~b}$ and $2 \mathrm{c}$ respectively.

Fig.1 shows the spring mass damper system with three degrees of freedom for model 1. The force F2 acts on mass $\mathrm{m} 2$ and the energy is transferred to other masses through the springs and a part of energy is absorbed by dampers. Springs $\mathrm{k} 1$ and $\mathrm{k} 4$; and dampers $\mathrm{c} 1$ and $\mathrm{c} 4$ are attached to rigid surfaces. The spring mass damper system is represented by the following equations.

$\mathrm{m} 1 \ddot{\mathrm{x}} 1+(\mathrm{c} 1+\mathrm{c} 2) \dot{\mathrm{x}} 1-\mathrm{c} 2 \mathrm{x} 2+(\mathrm{k} 1+\mathrm{k} 2) \mathrm{x} 1-\mathrm{k} 2 \mathrm{x} 2=0$

$\mathrm{m} 2 \ddot{\mathrm{x}} 2+(\mathrm{c} 2+\mathrm{c} 3) \dot{\mathrm{x}} 2-\mathrm{c} 2 \dot{\mathrm{x}} 1-\mathrm{c} 3 \dot{\mathrm{x}} 3+(\mathrm{k} 2+\mathrm{k} 3) \mathrm{x} 2-$

$\mathrm{k} 2 \times 1-\mathrm{k} 3 \times 3=\mathrm{F} 2$

$\mathrm{m} 3 \ddot{\mathrm{x}} 3+(\mathrm{c} 3+\mathrm{c} 4) \dot{\mathrm{x}} 3-\mathrm{c} 3 \dot{\mathrm{x}} 2+(\mathrm{k} 3+\mathrm{k} 4) \mathrm{x} 3-\mathrm{k} 3 \mathrm{x} 2=0$

The above three governing equations can be represented in matrix form as

$$
\begin{aligned}
M & =\left[\begin{array}{ccc}
m 1 & 0 & 0 \\
0 & m 2 & 0 \\
0 & 0 & m 3
\end{array}\right] \\
C & =\left[\begin{array}{ccc}
c 1+c 2 & -c 2 & 0 \\
-c 2 & c 2+c 3 & -c 3 \\
0 & -c 3 & c 3+c 4
\end{array}\right] \\
K & =\left[\begin{array}{ccc}
k 1+k 2 & -k 2 & 0 \\
-k 2 & k 2+k 3 & -k 3 \\
0 & -k 3 & k 3+k 4
\end{array}\right] \\
F & =\left\{\begin{array}{c}
0 \\
F 2 \\
0
\end{array}\right\}
\end{aligned}
$$

$M \ddot{x}+C \dot{x}+K x=F$

$\left[\begin{array}{ccc}m 1 & 0 & 0 \\ 0 & m 2 & 0 \\ 0 & 0 & m 3\end{array}\right]\left\{\begin{array}{l}\ddot{x} 1 \\ \ddot{x} \ddot{2} \\ \ddot{x} 3\end{array}\right\}+$

$\left[\begin{array}{ccc}c 1+c 2 & -c 2 & 0 \\ -c 2 & c 2+c 3 & -c 3 \\ 0 & -c 3 & c 3+c 4\end{array}\right]\left\{\begin{array}{c}\dot{x} 1 \\ \dot{x} 2 \\ \dot{x} 3\end{array}\right\}+$

$\left[\begin{array}{ccc}k 1+k 2 & -k 2 & 0 \\ -k 2 & k 2+k 3 & -k 3 \\ 0 & -k 3 & k 3+k 4\end{array}\right]\left\{\begin{array}{l}x 1 \\ x 2 \\ x 3\end{array}\right\}=\left\{\begin{array}{c}0 \\ F 2 \\ 0\end{array}\right\}$ for model 1a,

$$
\left[\begin{array}{ccc}
k 1+k 2 & -k 2 & 0 \\
-k 2 & k 2+k 3 & -k 3 \\
0 & -k 3 & k 3+k 4
\end{array}\right]\left\{\begin{array}{l}
x 1 \\
x 2 \\
x 3
\end{array}\right\}=\left\{\begin{array}{c}
0 \\
F 2 . e^{-w t} \\
0
\end{array}\right\}
$$

for model $1 b$,

$\left[\begin{array}{ccc}k 1+k 2 & -k 2 & 0 \\ -k 2 & k 2+k 3 & -k 3 \\ 0 & -k 3 & k 3+k 4\end{array}\right]\left\{\begin{array}{l}x 1 \\ x 2 \\ x 3\end{array}\right\}=\left\{\begin{array}{c}0 \\ F 2 . \delta(t) \\ 0\end{array}\right\}$

$\delta(t)=\left\{\begin{array}{l}1 \text { for } t \leq T \\ 0 \text { for } t>T\end{array}\right.$

for model 1c, respectively.

Fig. 2 shows the spring mass damper system with three degrees of freedom for model 2. The force F2 acts on mass $\mathrm{m} 2$ and the energy is transferred to other masses through the springs and a part of energy is absorbed by dampers c3, c4 and c5. Springs $\mathrm{k} 1$ and $\mathrm{k} 4$; and dampers $\mathrm{c} 4$ and $\mathrm{c} 5$ are attached to rigid surfaces. The spring mass damper system is represented by the following equations.

$\mathrm{m} 1 \ddot{x} 1+\mathrm{c} 5 \dot{\mathrm{x}} 1+(\mathrm{k} 1+\mathrm{k} 2) \mathrm{x} 1-\mathrm{k} 2 \mathrm{x} 2=0$

$\mathrm{m} 2 \mathrm{x} 2+\mathrm{c} 3 \dot{\mathrm{x}} 2-\mathrm{c} 3 \mathrm{x} 3+(\mathrm{k} 2+\mathrm{k} 3) \mathrm{x} 2-\mathrm{k} 2 \mathrm{x} 1-\mathrm{k} 3 \mathrm{x} 3=\mathrm{F} 2$

$\mathrm{m} 3 \ddot{\mathrm{x}} 3+(\mathrm{c} 3+\mathrm{c} 4) \dot{\mathrm{x}} 3-\mathrm{c} 3 \dot{\mathrm{x}} 2+(\mathrm{k} 3+\mathrm{k} 4) \mathrm{x} 3-\mathrm{k} 3 \mathrm{x} 2=0$

The above three governing equations can be represented in matrix form as

$M=\left[\begin{array}{ccc}m 1 & 0 & 0 \\ 0 & m 2 & 0 \\ 0 & 0 & m 3\end{array}\right]$

$C=\left[\begin{array}{ccc}c 5 & 0 & 0 \\ 0 & c 3 & -c 3 \\ 0 & -c 3 & c 3+c 4\end{array}\right]$

$K=\left[\begin{array}{ccc}k 1+k 2 & -k 2 & 0 \\ -k 2 & k 2+k 3 & -k 3 \\ 0 & -k 3 & k 3+k 4\end{array}\right]$

$F=\left\{\begin{array}{c}0 \\ F 2 \\ 0\end{array}\right\}$

$M \ddot{x}+C \dot{x}+K x=F$

$\left[\begin{array}{ccc}m 1 & 0 & 0 \\ 0 & m 2 & 0 \\ 0 & 0 & m 3\end{array}\right]\left\{\begin{array}{l}\ddot{x} 1 \\ \ddot{x} 2 \\ \ddot{x} 3\end{array}\right\}+\left[\begin{array}{ccc}c 5 & 0 & 0 \\ 0 & c 3 & -c 3 \\ 0 & -c 3 & c 3+c 4\end{array}\right]\left\{\begin{array}{l}\dot{x} 1 \\ \dot{x} 2 \\ x 3\end{array}\right\}+$

$\left[\begin{array}{ccc}k 1+k 2 & -k 2 & 0 \\ -k 2 & k 2+k 3 & -k 3 \\ 0 & -k 3 & k 3+k 4\end{array}\right]\left\{\begin{array}{l}x 1 \\ x 2 \\ x 3\end{array}\right\}=\left\{\begin{array}{c}0 \\ F 2 \\ 0\end{array}\right\}$ 
for model $2 \mathrm{a}$,

$\left[\begin{array}{ccc}m 1 & 0 & 0 \\ 0 & m 2 & 0 \\ 0 & 0 & m 3\end{array}\right]\left\{\begin{array}{l}\ddot{x} \ddot{1} \\ \ddot{x} 2 \\ \ddot{x} 3\end{array}\right\}+\left[\begin{array}{ccc}c 5 & 0 & 0 \\ 0 & c 3 & -c 3 \\ 0 & -c 3 & c 3+c 4\end{array}\right]\left\{\begin{array}{c}\dot{x} 1 \\ \dot{x} 2 \\ \dot{x} 3\end{array}\right\}+$
$\left[\begin{array}{ccc}k 1+k 2 & -k 2 & 0 \\ -k 2 & k 2+k 3 & -k 3 \\ 0 & -k 3 & k 3+k 4\end{array}\right]\left\{\begin{array}{l}x 1 \\ x 2 \\ x 3\end{array}\right\}=\left\{\begin{array}{c}0 \\ F 2 . e^{-w t} \\ 0\end{array}\right\}$

for model $2 b$,

$\left[\begin{array}{ccc}m 1 & 0 & 0 \\ 0 & m 2 & 0 \\ 0 & 0 & m 3\end{array}\right]\left\{\begin{array}{l}\ddot{x} \ddot{1} \\ \ddot{x} 2 \\ \ddot{x} \ddot{3}\end{array}\right\}+\left[\begin{array}{ccc}c 5 & 0 & 0 \\ 0 & c 3 & -c 3 \\ 0 & -c 3 & c 3+c 4\end{array}\right]\left\{\begin{array}{c}\dot{x} 1 \\ \dot{x} 2 \\ \dot{x} 3\end{array}\right\}+$

$\left[\begin{array}{ccc}k 1+k 2 & -k 2 & 0 \\ -k 2 & k 2+k 3 & -k 3 \\ 0 & -k 3 & k 3+k 4\end{array}\right]\left\{\begin{array}{l}x 1 \\ x 2 \\ x 3\end{array}\right\}=\left\{\begin{array}{c}0 \\ F 2 . \delta(t) \\ 0\end{array}\right\}$

$\delta(t)=\left\{\begin{array}{l}1 \text { for } t \leq T \\ 0 \text { for } t>T\end{array}\right.$

for model $2 \mathrm{c}$, respectively.

The spring mass damper systems with three degrees of freedom are modelled for two cases. The cases are represented in the Fig. 1 and Fig.2. The respective equation are solved for all the three type of external forces, that is, constant force, exponentially decaying force and the partial load over a time period are solved. The equations are solved using MATLAB. The conditions for which these models are solved for a loss factor of 0.15 are given in Table 1 .

TABLE -1: Conditions used for the simulation for two spring-mass-damper models

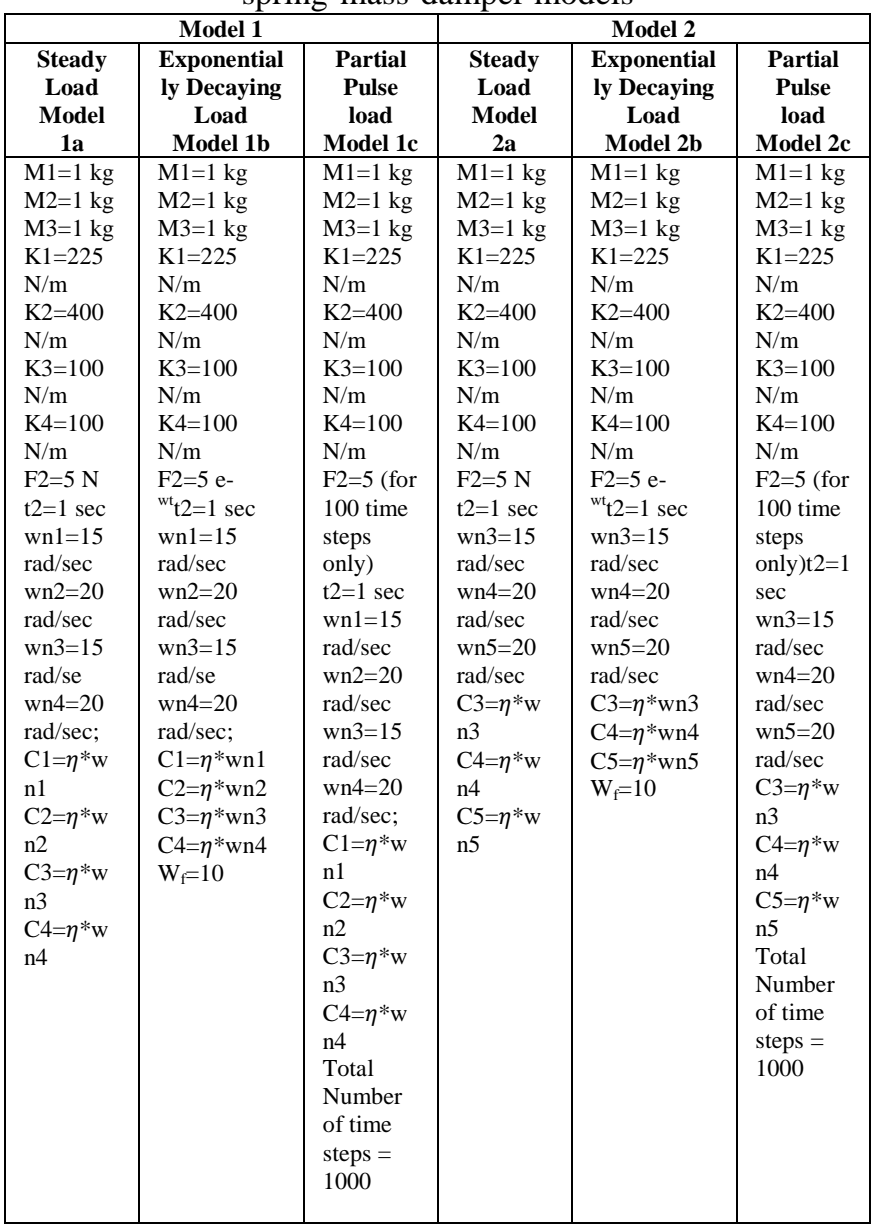

$\eta=0.15$, where $\eta$ is the coupling factor.

\section{SIMULATION RESULTS}

Based on the solutions, the displacements and velocities of the oscillators are obtained and the energies stored in the oscillators are calculated. Initially, for model 1, the three sub models are solved representing the cases of three different types of external loads as mentioned above. These sub models, namely, model $1 \mathrm{a}$, model $1 \mathrm{~b}$ and model $1 \mathrm{c}$ have been solved for displacement and velocities of the oscillators 1, 2 and 3. The displacements of oscillators are provided in Figs. 3 to 5 for model 1. Similarly, the displacements of oscillators are provided in Figs. 6 to 8 for model 2.

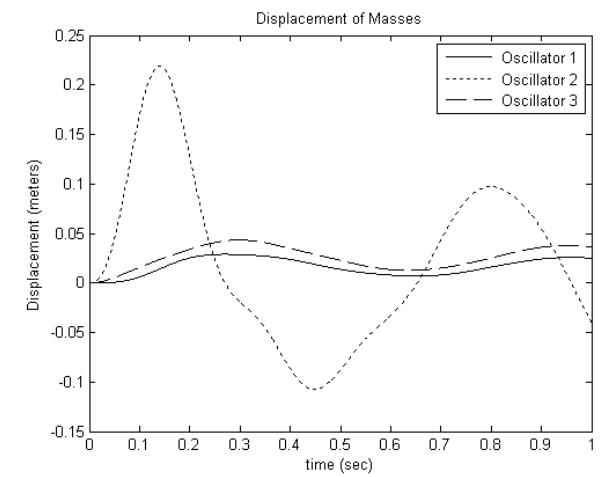

Fig -3: Displacement of Oscillators for Model 1a

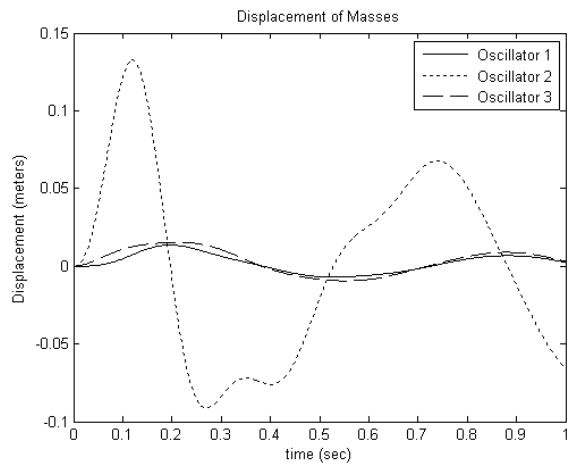

Fig -4: Displacement of Oscillators for Model 1b

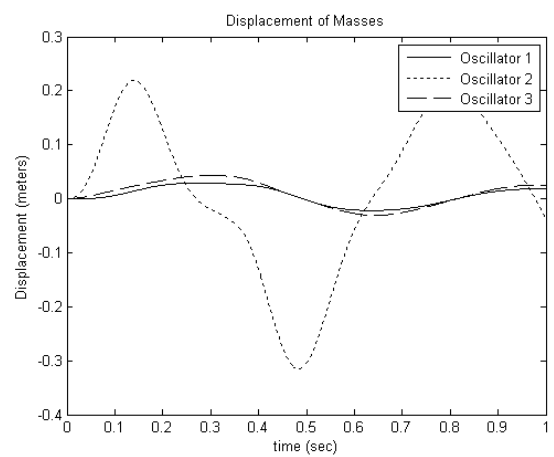

Fig -5: Displacement of Oscillators for Model 1c

Fig. 3 shows the maximum displacement is $0.22 \mathrm{~m}$ at 0.15 sec for oscillator 2 in case of a constant load and the displacement becomes $0.13 \mathrm{~m}$ when the constant load is replaced by the exponentially decaying load. When the load is applied for only $10 \%$ of the time period, the displacement again jumps to $0.22 \mathrm{~m}$. A difference one can notice between 
the models 1a and 1c, that the displacement are larger in case of partial load of $10 \%$ of the time period than the load acting on it for the entire time period, i.e. constant load. This is due to the fact that there is no controlling load on the oscillator 2 in case of model 1c for the remaining $90 \%$ of the time period and hence the displacements are high. It can also be concluded that more potential energy will be dissipated in case of model 1c than model 1a or model $1 \mathrm{~b}$.

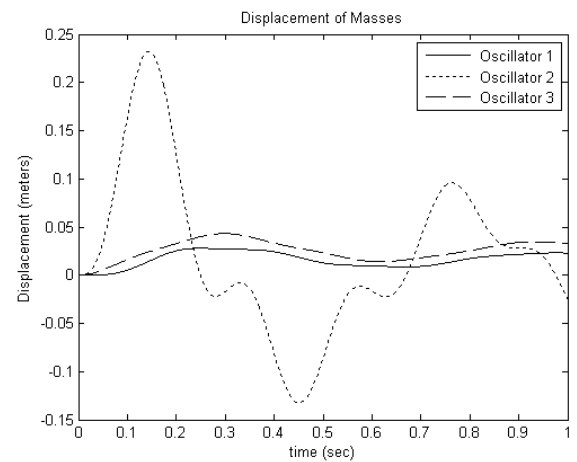

Fig -6: Displacement of Oscillators for Model 2a

By adding the damper $\mathrm{c} 5$ and removing $\mathrm{c} 1$ and $\mathrm{c} 2$ in model $2 \mathrm{a}$, the peak displacement of oscillator 2 has slightly increased from 0.22 meters to 0.23 , and the oscillations in displacement profile are more. This is due to the fact that two dampeners $\mathrm{c} 1$ and $\mathrm{c} 2$ are replaced with a single dampener. Even for the exponentially decaying load, model $2 \mathrm{~b}$ exhibits higher displacement than model $1 \mathrm{~b}$. All these behaviours can be attributed to the replacing two dampeners $\mathrm{c} 1$ and $\mathrm{c} 2$ in model 1 with one dampener $\mathrm{c} 5$ in model 2 . The displacement at $0.8 \mathrm{sec}$ in case of model $2 \mathrm{c}$ is again higher than that of model 1a. The reason behind this kind of behaviour is already explained above.

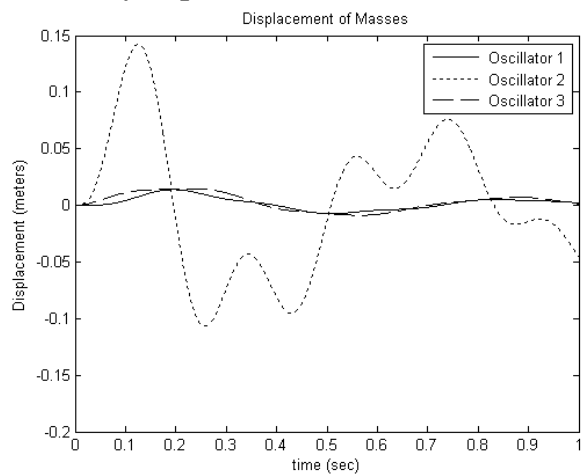

Fig -7: Displacement of Oscillators for Model 2b

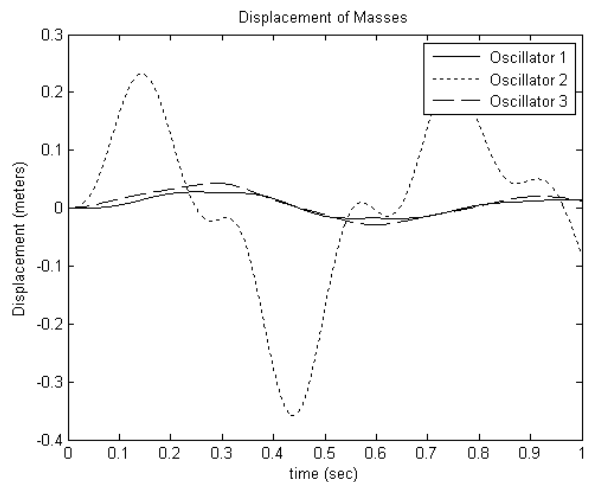

Fig -8: Displacement of Oscillators for Model 2c The dampeners in this case are modelled for loss factor of 0.15 . Although there is a minor variation in displacements for oscillators 1 and 3, their profiles remain almost same in all the two models. This is due to the fact that the excitation force is acting on oscillator 2.Overall there is significant change in the profiles of displacements between model 1 and model 2

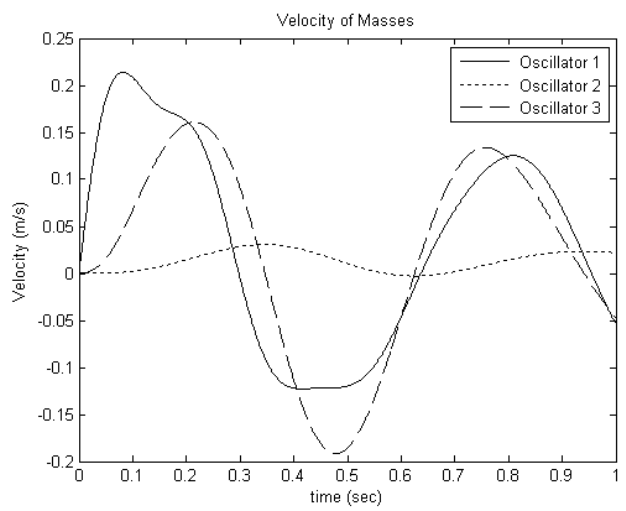

Fig -9:Velocity of Oscillators for Model 1a

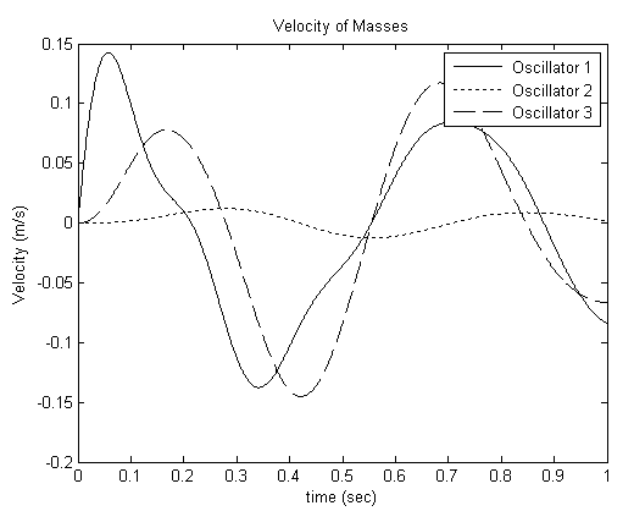

Fig -10:Velocity of Oscillators for Model 1b

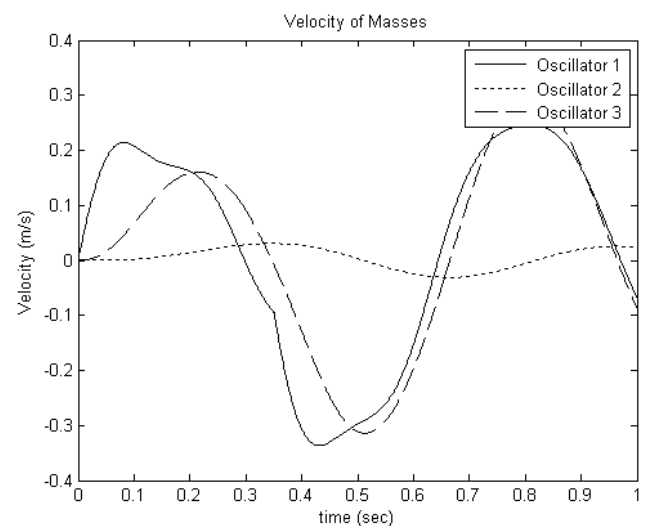

Fig -11: Velocity of Oscillators for Model 1c

Figs. 9 to 11 show the velocity of oscillators for the three sub models of model 1 and Figs 12 to 14 represent the velocity of oscillators for the three sub models of model 2 . It can be observed that the higher velocities occur for the oscillator 1 and 3 as the force is acting directly on oscillator 2 and there is no controlling load on oscillators 1 and 3 . Velocities of the oscillator 2 are very low compared to 
oscillators 1 and 3. By removing the damper $\mathrm{c} 1$ and $\mathrm{c} 2$, and adding the dampener $\mathrm{c} 5$, the peak velocity does not change much except those velocities near $0.75 \mathrm{sec}$. At the time step of $0.8 \mathrm{sec}$, the velocity of the oscillator 1 in model $1 \mathrm{c}$ is higher than that of the model $1 \mathrm{a}$, which means model 1c dissipates more kinetic energy than that of model 1a. Similarly, model 2 also has similar behaviours for all the three different external load types.

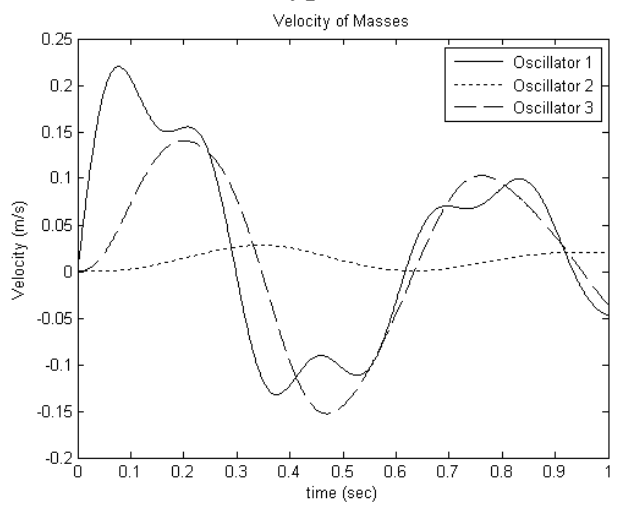

Fig -12: Velocity of Oscillators for Model 2a

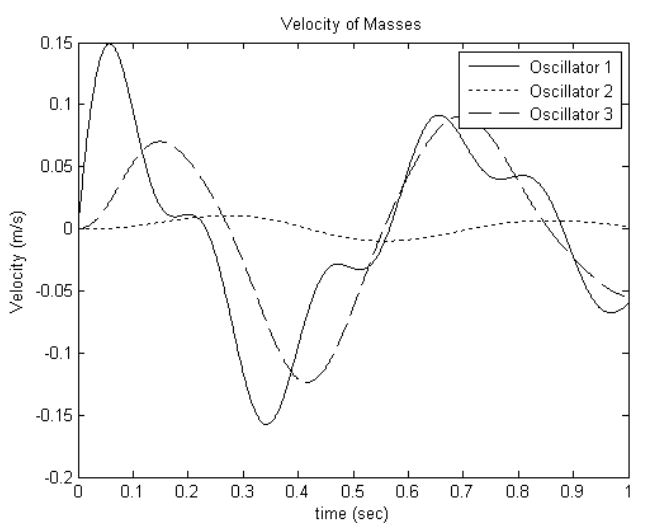

Fig -13: Velocity of Oscillators for Model 2b

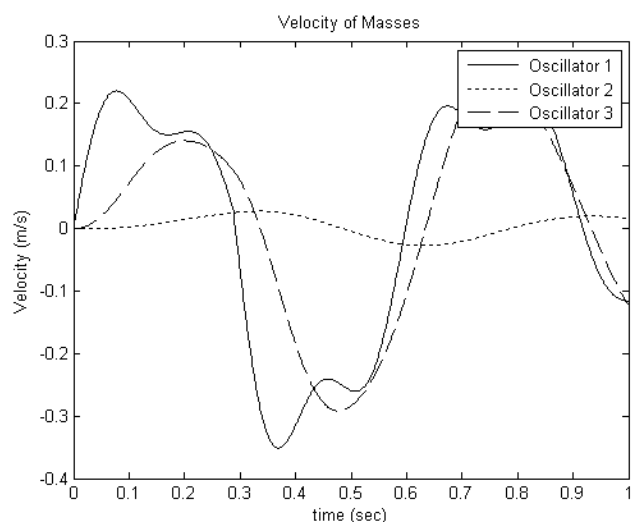

Fig -14: Velocity of Oscillators for Model 2c

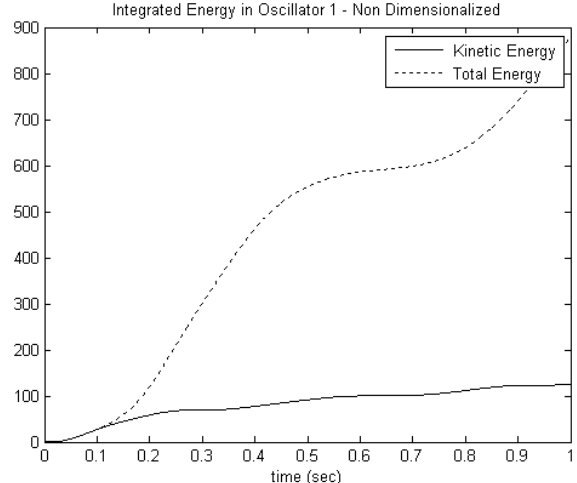

Fig -15: Integrated Energy in Oscillator 1 - NonDimensionalized for Modella

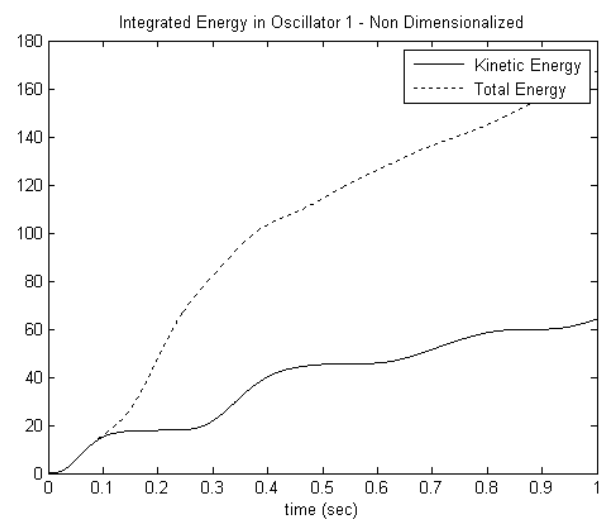

Fig -16 :Integrated Energy in Oscillator 1 - NonDimensionalized for Model 1b

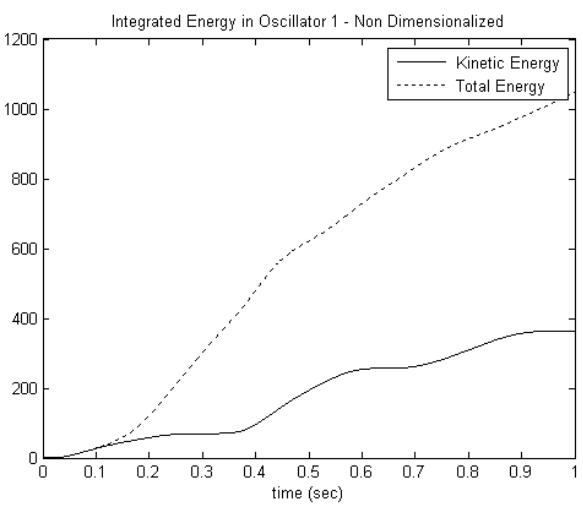

Fig -17: Integrated Energy in Oscillator 1 - NonDimensionalized for Model 1c 


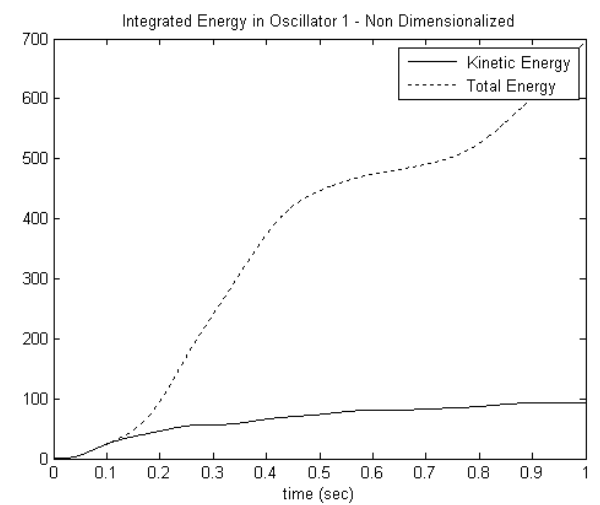

Fig -18: Integrated Energy in Oscillator 1 - NonDimensionalized for Model 2a

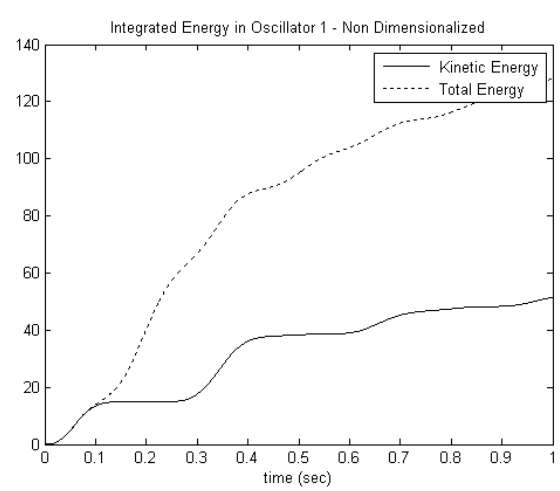

Fig -19: Integrated Energy in Oscillator 1 NonDimensionalized for Model 2b

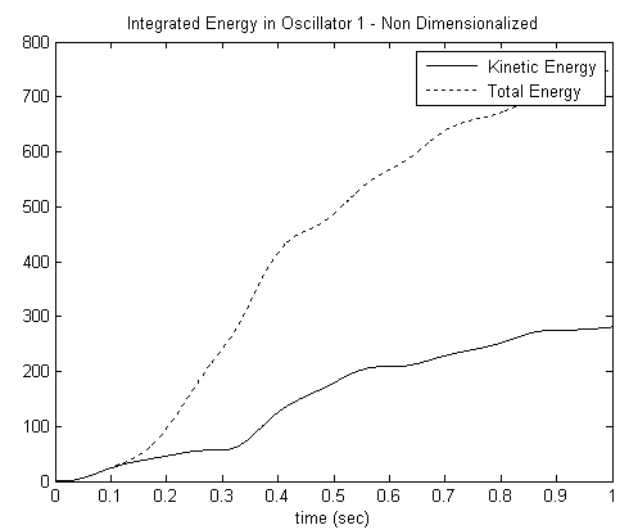

Fig -20: Integrated Energy in Oscillator 1 NonDimensionalized for Model 2c

Overall, from the plots it can be concluded that the addition or deletion of dampeners have little effect on the velocity of the oscillators compared to as it is experienced in the case of displacement of oscillators.

Figs. 15 to 20 show the integrated energy in the oscillator 1 for the models 1 and 2. Total energy dissipated in model 1 is less than that of model 2. In model 1a, it is 880 and in case of model $2 \mathrm{a}$, it is $680 \mathrm{in}$ a time period of $1 \mathrm{sec}$. As explained above, there are only 3 dampeners in model 2 a compared to model 1a, which has got 4 dampeners. The kinetic energy dissipation is almost same in both models. However, the energies dissipated is quite different when the type of external loads change.

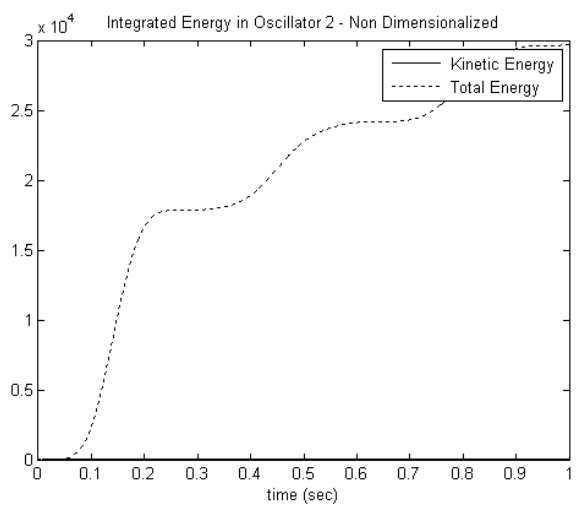

Fig -21: Integrated Energy in Oscillator 2 NonDimensionalized for Model 1a

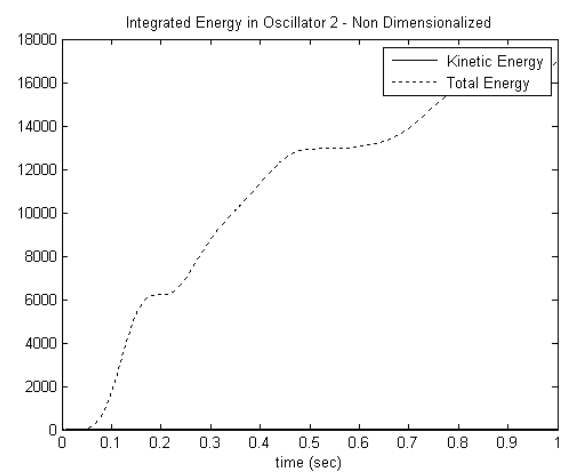

Fig -22: Integrated Energy in Oscillator 2 NonDimensionalized for Model 1b

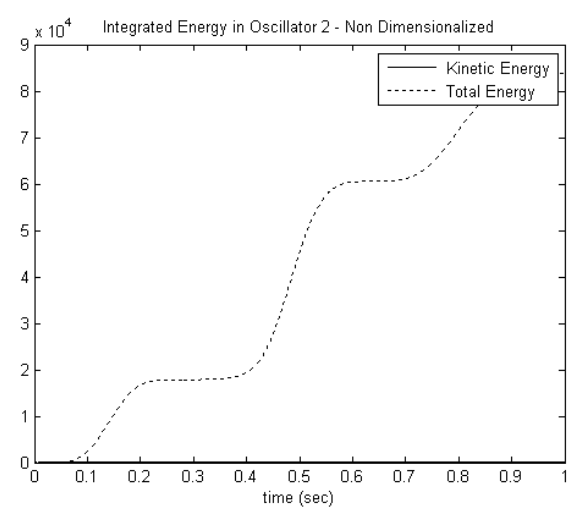

Fig -23: Integrated Energy in Oscillator 2 NonDimensionalized for Model 1c 


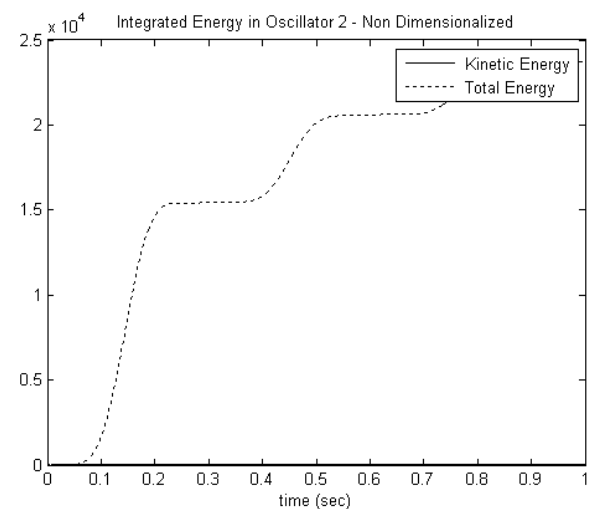

Fig -24: Integrated Energy in Oscillator 2 NonDimensionalized for Model 2a

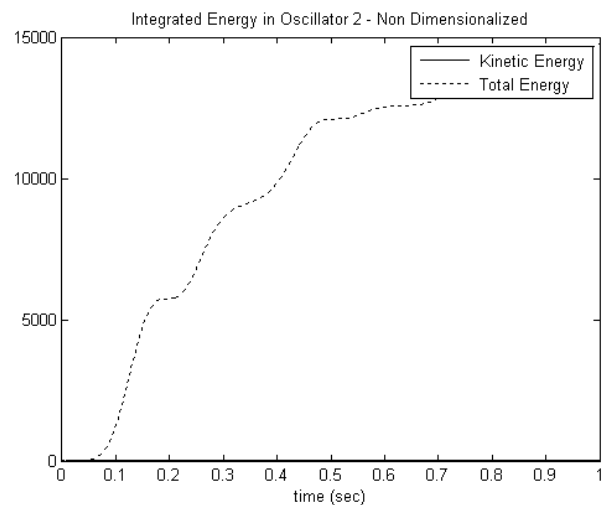

Fig -25: Integrated Energy in Oscillator 2 NonDimensionalized for Model 2b

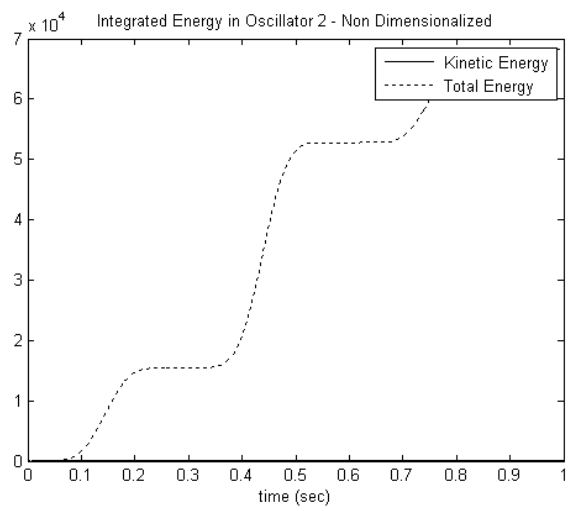

Fig -26: Integrated Energy in Oscillator 2 NonDimensionalized for Model 2c

Figs. 21 to 26 show the integrated energy for oscillator 2 . One interesting observation can be made between the integrated energy diagrams for oscillators 1 and 3 on one hand and oscillator 2 on other hand is, the component of kinetic energy in total energy is very minimal for oscillator 2 , which means the most of potential energy is present in the total energy. This is due to the fact that the load directly acts on the oscillator and the corresponding energy is dissipated in overcoming the stiffness of the system.

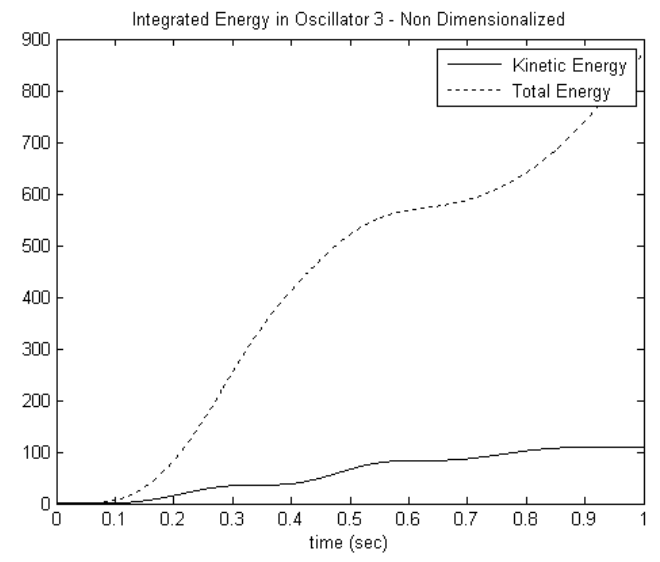

Fig -27: Integrated Energy in Oscillator 3 NonDimensionalized for Model 1a

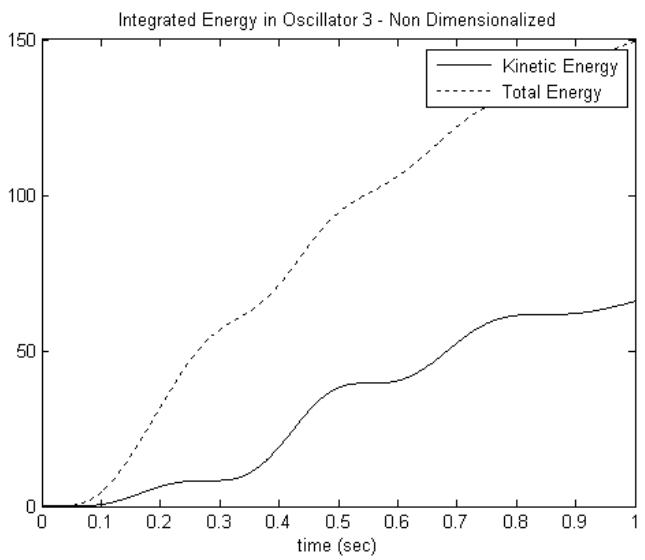

Fig -28: Integrated Energy in Oscillator 3 NonDimensionalized for Model $1 \mathrm{~b}$

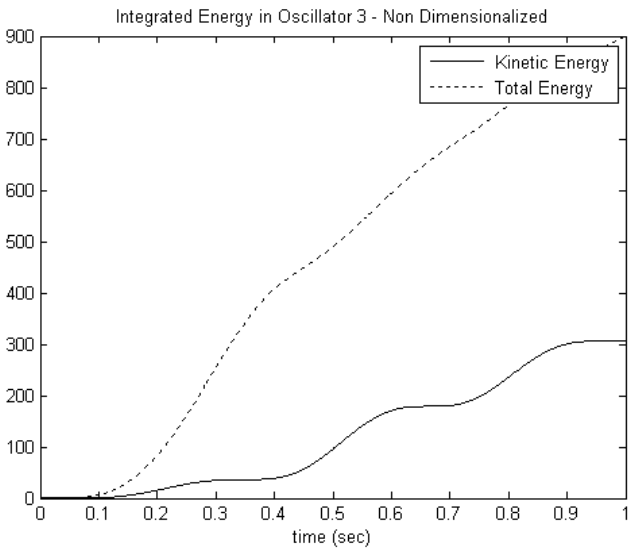

Fig -29: Integrated Energy in Oscillator 3 - NonDimensionalized for Model 1c

One can also notice from the Figs. 21 to 26 that, as the load is changed from a steady state to aexponentially decaying load, the dissipated energy also reduces. But when a load is applied over a partial time period, more energy is dissipated than the steady state case due to the reasons mentioned in the above paragraphs. 


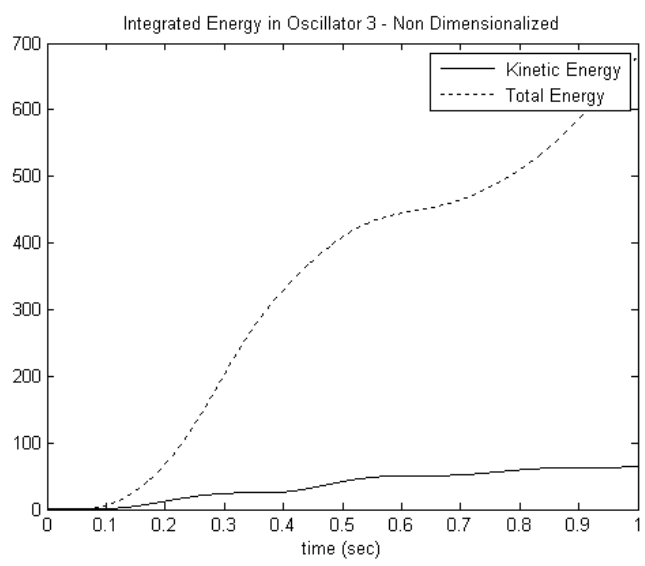

Fig -30: Integrated Energy in Oscillator 3 NonDimensionalized for Model 2a

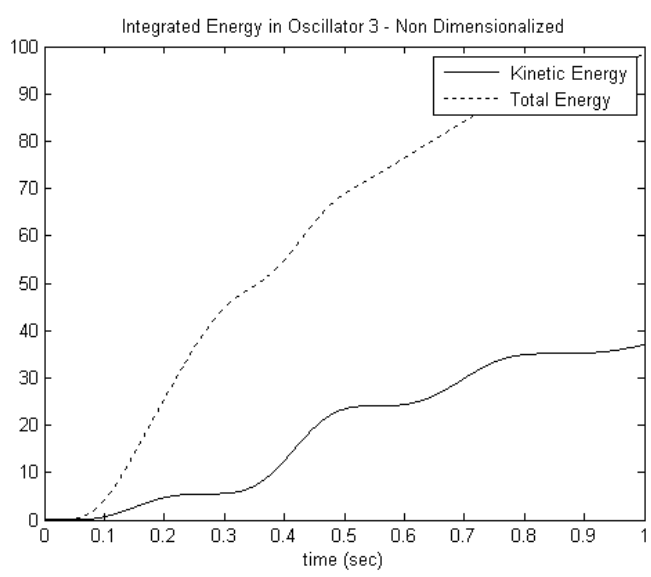

Fig -31: Integrated Energy in Oscillator 3 NonDimensionalized for Model $2 \mathrm{~b}$

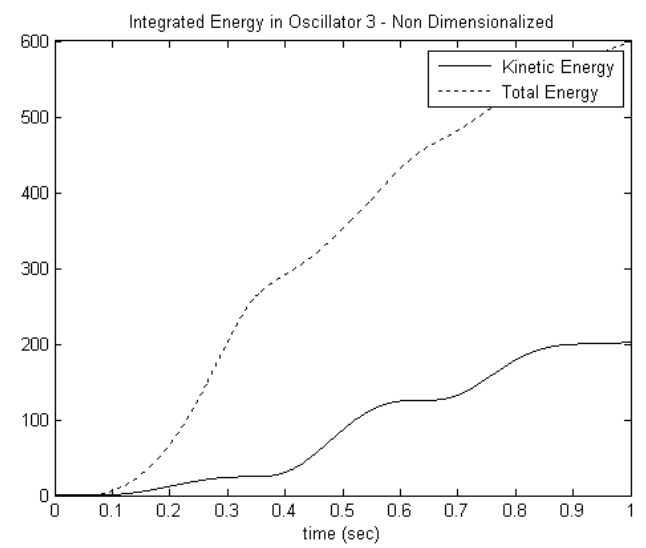

Fig -32: Integrated Energy in Oscillator 3 - NonDimensionalized for Model 2c

Figs. 27 to 32 show the corresponding non dimensionalized integrated energies for the oscillator 3 for models 1 and 2. In contrast to the integrated energies of the oscillator 2 , here there is a significant contribution of kinetic energy in all the cases of external load.

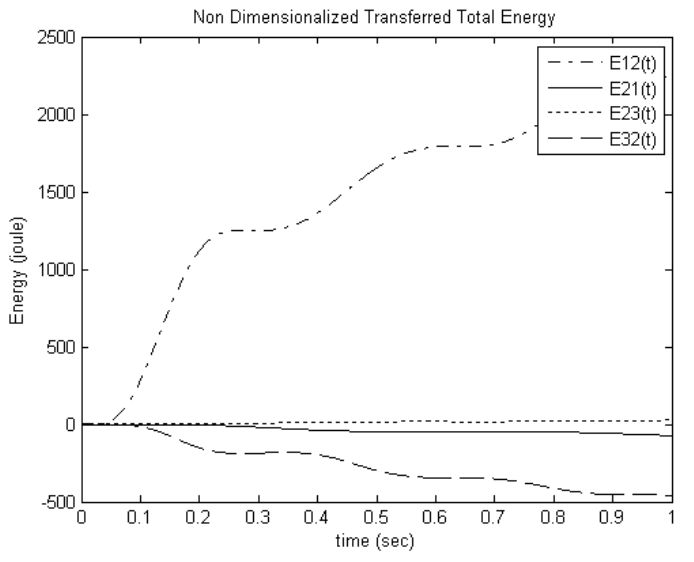

Fig - 33: Transferred Total Energy - Non-Dimensionalized for Model 1a

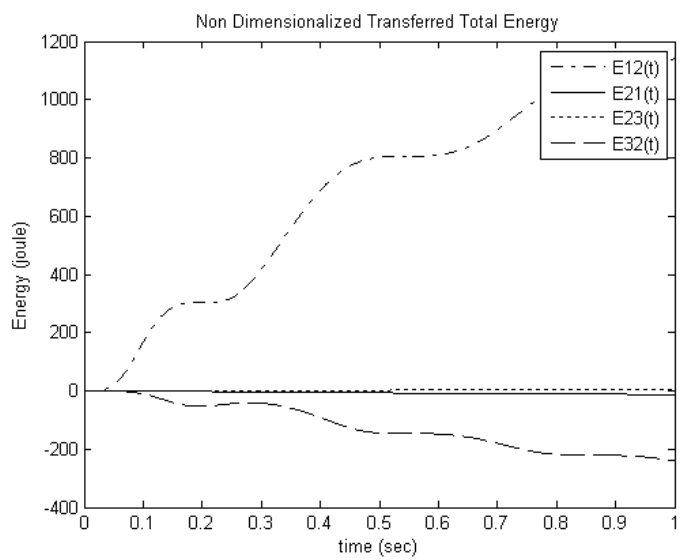

Fig -34: Transferred Total Energy - Non-Dimensionalized for Model 1b

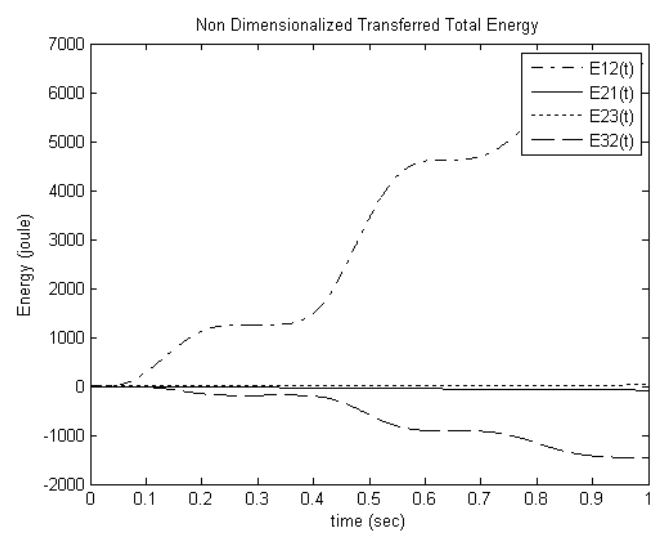

Fig - 35: Transferred Total Energy - NonDimensionalized for Model 1c 


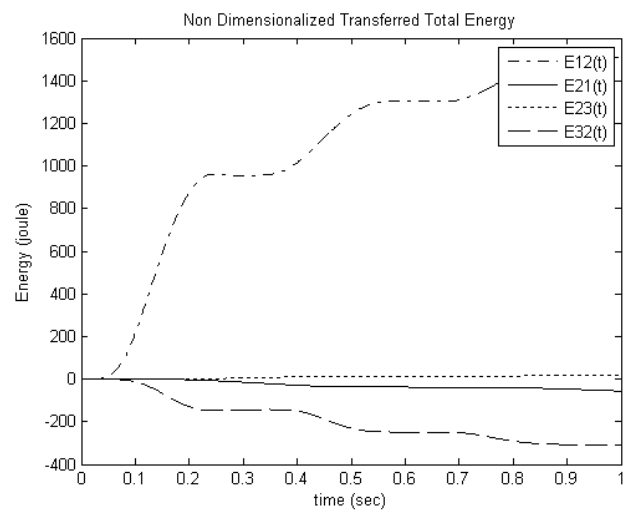

Fig - 36: Transferred Total Energy - Non-Dimensionalized for Model 2a

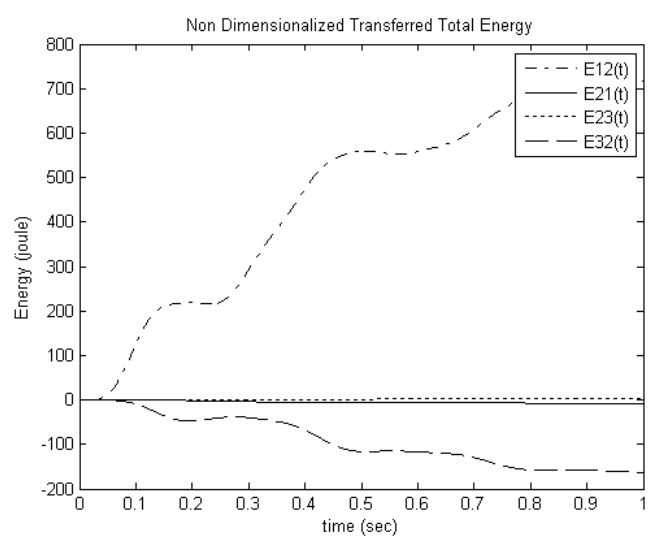

Fig - 37: Transferred Total Energy - Non -Dimensionalized for Model 2b

Figs. 32 to 38 show the transferred total energy for models 1 and 2 and their sub models of different external loads. Highest energy is transferred from oscillator 1 to 2 and next highest from 3 to 2 in case of load over a partial time period than the other two cases. Negative energy means energy flows in the reverse direction.

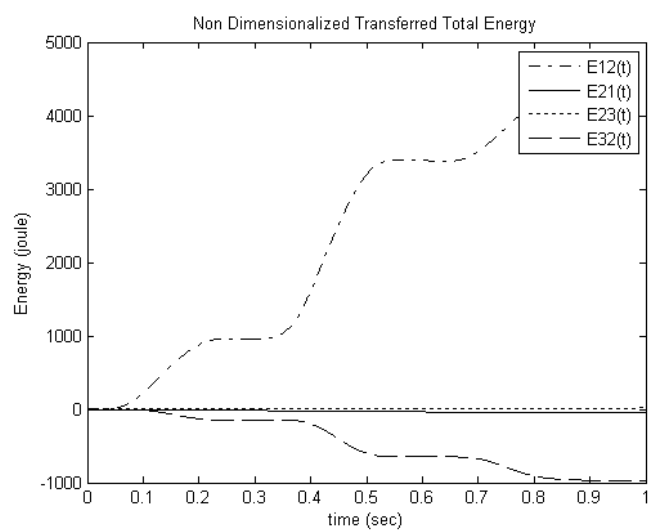

Fig -38: Transferred Total Energy - Non-Dimensionalized for Model 2c

\section{CONCLUSION}

Spring mass dampeners with three degrees of freedom are modeled for two cases. The external loads applied on the oscillator 2 is varied to three types, namely, constant, exponentially decaying and partial load over a time period. The equations are solved numerically and, the displacements and integrated energies are computed for the three oscillators.Based on the study of the behavior of these models, for a loss factor of 0.15 , by adding the damper c5 and removing $\mathrm{c} 1$ and $\mathrm{c} 2$ in model $2 \mathrm{a}$ the displacement of the oscillatorincreases while the oscillations in profile of the displacement also increases. The two effects are due to reducing the total number of dampeners from 4 to 3 in models 1 to 2 . There is significant change in the velocities of oscillator 2 for this change in the number of dampeners. However, when the load is changed from full load to a partial load over a time period, there is significant increase in the displacement and the velocity at near $0.75 \mathrm{sec}$, which means it dissipates more energy. The contribution of the kinetic energy is minimal for oscillator 2 in all cases and the total energy is constituted mostly of potential energy. However, there is substantial contribution both by potential as well as kinetic energy for oscillators 1 and 3 .

\section{REFERENCES}

[1] Harris and Creede: Shock and vibration handbook, 1996.

[2] Ayre, R. S.: Engineering vibrations, 1958

[3] Timoshenko S, Young D H, Weaver W.: Vibration problems in engineering, 1974

[4] Snowdon J C.: Vibration and shock in damped mechanical systems, 1968

[5] Morrow: Shock and vibration engineering, 1963

[6] Chu, F.H., and Wang, B.P., " Experimental Determination of Damping in Materials and Structures," Damping Application for Vibration Control, Torvik,P.J., editor, ASME Winter Annual Meeing, Chicago, 1980, pp. 113-122.

[7] Carfagni, M. and Pierini, M. "Determining the Loss Factor by the Power Input Method (PIM), Part 1: Numerical Investigation." Journal of Vibration and Acoustics, 121 1997, pp. 417-421.

[8] Bies, D.A. and Hamid, S. "In Situ Determination of Loss and Coupling Loss Factors by the Power Injection Method," Journal of Sound and Vibration, 70(2), 1980, pp. 187-204.

[9] Polytec OFV-3000/OFV-302 Vibrometer Operators Manual, 1993, Waldbronn, Germany.

[10] Silva, J.M.M., Maia, N.M.M. and Ribeiro, A.M.R., "Cancellation of Mass-Loading Effects of Transducers and Evaluation of Unmeasured Frequency Response Functions," Journal of Sound and Vibration, 236(5), 2000, pp. 761-779.

[11] Ashory, M.R.. "Correction of Mass-Loading Effects of transducers and Suspension Effects in Modal Testing," 1998 Proceedings of the XVIth IMAC, CA, U.S.A, pp. 815-828 
[12] H. Dande, "Homework \#2, AE 790, Structural Acoustics", spring, 2009.

[13] W. Liu and M. S. Ewing, "Experimental and Analytical Estimation of Loss Factors by the Power Input Method," AIAA Journal, vol. 45, No. 2, pp. 477484, 2007.

[14] H. Dande, "Panel Damping Loss Factor Estimation using the Random Decrement Technique," Master of Science, Aerospace Engineering, University of Kansas,Lawrence, 2009.

[15] M. S. Ewing, et al., "Validation of Panel Damping Loss Factor Estimation Algorithms Using a Computational Model," presented at the 50th AIAA/ASME/ASCE/AHS/ASC structures Structural Dynamics and Materials Conference, 2009.

[16] K. Vatti, "Damping Estimation of Plates for statistical Energy Analysis," Master of Science, Aerospace Engineering, University of Kansas, Lawrence, 2010.

[17] J. He and Z.-F. Fu, "Modal Analysis," ButterworthHeinemann, 2001.

[18] B. Bloss and M. D. Rao, "Measurement of Damping In Structures by the power input method," Experimental Techniques, vol. 26, pp. 30-33, 2002.

[19] M. L. Lai and A. Soom, "Prediction of Transient Vibration Envelopes Using Statistical Energy Analysis Techniques," Journal of Vibration and Acoustics, vol. 112, pp. 127-137, 1990.

[20] F.H. Chu, and B.P. Wang, "Experimental Determination of Damping in Materials and Structures," Damping Application for Vibration Control, Torvik,P.J., editor, ASME Winter Annual Meeting, Chicago, 1980, pp. 113-122.

[21] M. Carfagni and M. Pierini, "Determining the Loss Factor by the Power Input Method (PIM), Part 1: Numerical Investigation." Journal of Vibration and Acoustics, 121 1997, pp. 417-421.

[22] D.A. Bies and S. Hamid "In Situ Determination of Loss and Coupling Loss Factors by the Power Injection Method," Journal of Sound and Vibration, 70(2), 1980, pp. 187-204.

[23] [18] W. Pawlus, J.E. Nielsen, H.R. Karimi, and K.G. Robbersmyr, "Mathematical modeling and analysis of a vehicle crash", The $4^{\text {th }}$ European Computing Conference, Bucharest, Romania, April 2010.

[24] W. Pawlus, J.E. Nielsen, H.R. Karimi, and K.G. Robbersmyr, "Development of mathematical models for analysis of a vehicle crash," WSEAS Transactions on Applied and Theoretical Mechanics, vol.5, No.2, pp.156-165, 2010.

[25] W. Pawlus, J.E. Nielsen, H.R. Karimi, and K.G. Robbersmyr, "Further results on mathematical models of vehicle localized impact," The $3^{\text {rd }}$ International Symposium on Systems andControl in Aeronautics and Astronautics, Harbin, China, June2010.

[26] H.R. Karimi and K.G. Robbersmyr, "Wavelet-based signal analysis of a vehicle crash test with a fixed safety barrier," WSEAS $4^{\text {th }}$ European Computing Conference, Bucharest, Romania, April 20-22, 2010.

\section{BIOGRAPHIES}

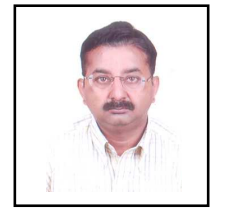

J.O. Kiran is having total twelve years of experience in teaching Mechanical and Machine Design Engineering subjects. $\mathrm{He}$ is working in the area of vibration analysis and design engineering. He has industrial experience in the field of Hydraulics. He has supervised 16 undergraduate projects and 03 post graduate projects. He has presented many research papers in the international and national conference and journals.

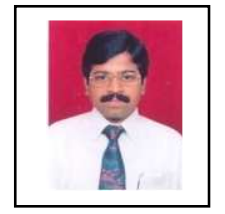

Dr. D. Shivalingappa received Ph.D degree from IIT Roorkee India. He is working in the area of Vibration Analysis, Design Engineering Composite Materials. He has supervised 9 postgraduate projects and 29 undergraduate projects. Currently he is supervising $3 \mathrm{Ph} . \mathrm{D}$ projects and 4 postgraduate projects. He has published 21 research papers in journal/ conferences of national and international repute. 
